\title{
Câncer cervical e de mama em pacientes com lúpus eritematoso sistêmico
}

\author{
Breast and cervical cancer in patients with systemic lupus erythematosus
}

Artigo Original

Lúpus eritematoso sistêmico/complicações

Neoplasias

Colo do útero/patologia

Neoplasias do colo do útero/complicações

Neoplasias da mama/complicações

Keywords

Lupus erythematosus, systemic/complications

Neoplasms

Cervix uteri/pathology

Uterine cervical neoplasms/complications Breast neoplasms/complications

\section{Resumo}

OBJETIVO: Estudar a frequência de neoplasias em uma amostra da população brasileira feminina com lúpus eritematoso sistêmico. MÉTODOS: Este é um trabalho retrospectivo de revisão de prontuários de 395 mulheres com lúpus eritematoso sistêmico, diagnosticadas pela presença de, pelo menos, 4 dos critérios classificatórios do Colégio Americano de Reumatologia para diagnóstico dessa doença e acompanhadas nos últimos 10 anos em um ambulatório especializado em Reumatologia. Foram coletados dados demográficos (idade e etnia das pacientes), dados acerca de duração de doença, de uso dos principais imunomoduladores e de diagnóstico de neoplasias. As informações obtidas foram tabuladas em tabelas de frequência e de contingência. A taxa de incidência de neoplasias nas mulheres com lúpus foi comparada com a da população em geral para a mesma região demográfica, nos últimos dez anos, utilizando, para isso, dados publicados pelo Instituto Nacional de Câncer (INCA). A associação dos dados foi feita pelos testes de Fisher e do $\chi^{2}$, quando os dados eram nominais, e de Mann-Whitney, quando numéricos. Foi adotada a significância de 5\%. RESULTADOS: Foram identificados 22 casos de tumores malignos (22 casos/395 ou 5,5\% da amostra) sendo os mais comuns os de útero ( 10 casos/395 ou 2,5\% da amostra) e de mama (9 casos/395 ou $2,2 \%$ da amostra). A presença de tumores estava associada com tempo de duração de doença $(p=0,006)$, não sendo observada influência de tratamento com metotrexate $(p=0,1)$, azatioprina $(p=0,9)$, ciclofosfamida $(p=0,6)$ e glicocorticoides $(p=0,3)$. Os tumores de mama e de colo do útero foram mais comuns no lúpus eritematoso sistêmico do que na população em geral ( $p<0,0001$ para ambos). CONCLUSÃO: Na presente amostra foi observada uma alta prevalência de tumores malignos, que foram mais comuns nas pacientes com maior tempo de doença. Os tumores mais frequentes foram os de mama e de útero, cuja incidência foi maior do que na população em geral. A presença de tumores não foi influenciada pelo uso de imunossupressores nem glicocorticoides

\section{Abstract}

PURPOSE: To study the incidence of tumors in a Brazilian sample of women with systemic lupus erythematosus. METHODS: This is a retrospective study of 395 medical charts from women with systemic lupus erythematosus diagnosed by the presence of at least 4 of the American College of Rheumatology classification criteria for the diagnosis of this disease and followed for the last 10 years in a rheumatology outpatient clinic. Demographic data (age and ethnicity of patients), data on disease duration, use of immunomodulators and on the presence of neoplasms were listed. Results are presented in frequency and contingency tables. The incidence rate of malignancies in women with lupus was compared with that of the general population for the same demographic region for the past ten years, using data published by the Brazilian National Cancer Institute (INCA). Association studies were carried out by the Fisher and $\chi^{2}$ tests, when the data were nominal, and by Mann-Whitney test, when numeric. The level of significance was set at $5 \%$. RESULTS: Twenty-two cases of malignant tumors were identified during these 10 years of follow-up (22 cases/395 or $5.5 \%$ of the sample), being the most common those of the uterine cervix ( 10 cases/395 or $2.5 \%$ of the sample) and breast cancer ( 9 cases/395 or $2.2 \%$ of the sample). The presence of tumors was associated with disease duration ( $p=0.006)$ and was not influenced by treatment with methotrexate $(p=0.1)$, azathioprine $(p=0.9)$, cyclophosphamide $(p=0.6)$ and glucocorticoids $(p=0.3)$. Breast and uterine cervix tumors were more common in systemic lupus erythematosus women than in the general population $(p<0.0001$ for both). CONCLUSION: A high prevalence of malignant tumors was found in this sample, with tumors being more common in patients with longer disease duration. The most frequent tumors affected the breast and uterus at a higher incidence than in the general population. The presence of tumors was not influenced by the use of glucocorticoids or immunosuppressors.
Correspondênnia

Thelma Larocca Skare

Rua Augusto Stellfeld, 1.908 - Bigoriliho

(EP: $80730-150$

Curitiba (PR), Brasi

Recebido

$16 / 06 / 2014$

Aceito com modificacōoes

$22 / 07 / 2014$

Serviço de Reumatologia do Hospital Universitário Evangélico de Curitiba - HUEC - Curitiba (PR), Brasil.

'Serviço de Reumatologia, Hospital Universitário Evangélico de Curitiba - HUEC; Curso de Medicina, Faculdade Evangélica do Paraná - Curitiba (PR), Brasil.

${ }^{2}$ Curso de Medicina, Faculdade Evangélica do Paraná - Curitiba (PR), Brasil.

Conflito de interesses: não há. 
Introdução

O lúpus eritematoso sistêmico (LES) é uma doença de autoimunidade com preferência pelo gênero feminino, atingindo uma proporção de dez mulheres para um homem $^{1}$. Tal fato se deve, pelo menos em parte, à atuação dos estrógenos, que favorecem a proliferação de linfócitos $\mathrm{T}$ e B, aumentam a expressão de moléculas de adesão e reduzem a apoptose de células B autorreativas, além de favorecerem uma resposta imune do tipo Th2 ${ }^{1}$.

Com o advento de meios de diagnóstico precoce e de opções terapêuticas mais eficientes, as pacientes com LES têm tido um melhor prognóstico. A sobrevida em 10 anos passou de menos de $50 \%$ em 1955 para mais de $90 \%$ recentemente ${ }^{2,3}$. Com isso, pode-se obter uma visão mais clara não só das lesões causadas pela doença e seu tratamento mas também de possíveis associações com outras entidades mórbidas, antes obscurecidas pela curta sobrevida.

Descreve-se que pacientes com LES têm maior prevalência de malignidades ressaltando-se, na literatura, a alta prevalência de tumores de linha linfoide e de colo do útero ${ }^{4,5}$. O aumento da sobrevida no LES pode favorecer o aparecimento desses tumores não só pelo envelhecimento gradual da população, como também pelo fato de que tais pacientes ficam expostas, por mais tempo, a um ambiente de desregulação imune. Estudos em humanos e em animais demonstram que o microambiente da maioria dos tumores é altamente imunossupressor ${ }^{6}$. O aumento no perfil de citocinas Th2, principalmente da interleucina 10 (IL-10), o que é próprio do LES, favorece a tolerância do organismo a células epiteliais infectadas por papilomavírus humano (HPV), facilitando a progressão do câncer de colo do útero ${ }^{7}$.

Muitas neoplasias, assim como o próprio lúpus, são influenciadas por fatores genéticos ${ }^{1,8,9}$. Sendo a população brasileira altamente miscigenada do ponto de vista étnico, ela apresenta um background genético peculiar, o que a torna única nesse aspecto ${ }^{10}$. Isso impede que muitas informações obtidas em populações mais homogêneas sejam válidas para o nosso contexto. $\mathrm{O}$ presente trabalho foi realizado com a finalidade de verificar a ocorrência de neoplasias em população de lúpus na região Sul do Brasil.

\section{Métodos}

Este trabalho foi aprovado pelo Comitê de Ética em Pesquisa: número 253.532/Sociedade Evangélica Beneficente de Curitiba, Paraná. Uma amostra de 395 pacientes do gênero feminino com LES em acompanhamento pelo ambulatório de reumatologia de um único hospital terciário nos últimos dez anos foi estudada. Para que as pacientes fossem incluídas, elas deveriam ter pelo menos 4 dos critérios do Colégio
Americano de Reumatologia para classificação de LES ${ }^{11}$ e diagnóstico dessa colagenose feito após os 16 anos. Foram excluídas as pacientes com lúpus juvenil e pacientes cujos prontuários estavam incompletos.

Este é um trabalho retrospectivo no qual dados demográficos, de perfil clínico e de tratamento da doença foram obtidos pela revisão dos prontuários. A pergunta acerca de aparecimento de tumores foi feita de maneira rotineira e anotada no prontuário, sendo os mesmos analisados até a obtenção desse dado.

A amostra estudada compunha-se de pacientes com diagnóstico do LES feito entre 16 e 88 anos de idade (mediana de 42 anos), com tempo de acompanhamento mediano de 105 meses (intervalo interquartil - IIQ 48-132 meses) e formada por $63,4 \%(167 / 263)$ de pacientes autodeclarados caucasianos e de 36,5\% (96/263) de pacientes autodeclarados afrodescendentes. As características clínicas, sorológicas e de tratamento dessa amostra estão na Tabela 1.

Para a comparação dos achados da presente amostra com a população geral, foram utilizados dados dos últimos dez anos de incidência das principais neoplasias (mama e de colo do útero) na mesma região geográfica, através de relatórios do Instituto Nacional de Câncer José de Alencar Gomes da Silva (INCA) ${ }^{12}$.

Os dados foram coletados em tabelas de frequência e de contingência. Medidas de tendência central foram expressas em média e desvio padrão (DP) quando a amostra era gaussiana, e mediana e intervalos interquartis nas não gaussianas. A normalidade de distribuição dos dados foi pesquisada pelo teste de Kolmogorov-Smirnov. Os testes de Fisher ou do $\chi^{2}$ foram usados para estudo $\mathrm{da}$ associação entre presença de tumores e variáveis nominais, como etnia e tipo de tratamento utilizado, assim como para comparação de incidência de neoplasias entre a amostra de LES e a população geral. O teste $t$ pareado ou de Mann-Whitney foi utilizado para a análise de associação de dados numéricos como os entre neoplasias com tempo de doença e com idade do paciente. Os testes foram calculados com auxílio do software Graph Pad Prism versão 5.0. A significância adotada foi de 5\%.

\section{Resultados}

$\mathrm{Na}$ amostra estudada, ocorreram 22/395 casos de tumores malignos diagnosticados após o aparecimento do LES (5,5\%). A distribuição da localização desses tumores pode ser encontrada na Tabela 2 , onde se observa a preponderância de tumores de mama e de colo do útero.

Não foram observadas diferenças no número de tumores de acordo com a idade (idade mediana de 45,0 anos em pacientes com tumores versus 42,0 anos nos sem tumores; $p=0,2$ ), nem raça (caucasianos com $n=157 / 244$ ou 64,3\% nos sem tumores e 10/19 ou $52,6 \%$ nos com tumores; $\mathrm{p}=0,3)$. O tempo 
Tabela 1. Análise descritiva do perfil clínico, sorológico e de tratamento da amostra de 395 pacientes femininas com lúpus eritematoso sistêmico

\begin{tabular}{|c|c|c|}
\hline & n/amostra & $\%$ \\
\hline \multicolumn{3}{|l|}{ Perfil clínico* } \\
\hline Erupção em borboleta & $204 / 366$ & 55,7 \\
\hline Lúpus discoide & $66 / 358$ & 18,4 \\
\hline Fotossensibilidade & $295 / 389$ & 75,8 \\
\hline Serosites (pleurite e/ou pericardite) & $74 / 384$ & 19,2 \\
\hline Anemia hemolítica & $26 / 390$ & 6,6 \\
\hline Leucopenia & $108 / 384$ & 28,3 \\
\hline Plaquetopenia & $91 / 377$ & 24,1 \\
\hline Psicose & 19/389 & 4,8 \\
\hline Convulsão & $41 / 392$ & 10,4 \\
\hline Glomerulonefrite** & $162 / 395$ & 41,0 \\
\hline Classe 6 & $11 / 162$ & \\
\hline Classe 5 & $29 / 162$ & \\
\hline Classe 4 & $66 / 162$ & \\
\hline Classe 3 & $34 / 162$ & \\
\hline Classe 2 & $22 / 162$ & \\
\hline \multicolumn{3}{|l|}{ Perfil de autoanticorpos } \\
\hline Fator antinuclear & $395 / 395$ & 100,0 \\
\hline Anticorpo anti-Ro & $132 / 364$ & 36,2 \\
\hline Anticorpo anti- La & $65 / 362$ & 17,9 \\
\hline Anticorpo anti-dsDNA & $118 / 370$ & 31,8 \\
\hline Anticorpo anti-RNP & $81 / 283$ & 28,6 \\
\hline Anticorpo anti-Smith & $79 / 358$ & 22,0 \\
\hline Anticardiolipina IgG & $55 / 372$ & 14,7 \\
\hline Anticardiolipina IgM & $43 / 368$ & 11,6 \\
\hline Anticoagulante lúpico & $46 / 312$ & 14,7 \\
\hline \multicolumn{3}{|l|}{ Perfil de tratamento*** } \\
\hline Antimaláricos & $322 / 395$ & 81,5 \\
\hline Azatioprina & $141 / 395$ & 35,6 \\
\hline Ciclofosfamida & $98 / 395$ & 24,8 \\
\hline Metotrexate & $81 / 395$ & 20,5 \\
\hline Prednisona & $259 / 395$ & 65,5 \\
\hline Talidomida & $14 / 395$ & 3,5 \\
\hline
\end{tabular}

*Um achado clínico foi considerado como positivo quando de acordo com os critérios classificatórios do Colégio Americano de Reumatologia para diagnóstico de LES ${ }^{11}$.

**todas as glomerulonefrites, exceto as da classe 6, possuíam comprovação por biópsia; dsDNA: DNA de dupla hélice; RNP: ribonucleoproteína; lgG: imunoglobulina G; $\lg M$ : imunoglobulina $M_{;}{ }^{*} * *$ o tratamento considerado foi o realizado desde 0 diagnóstico de lúpus eritematoso sistêmico até a data de diagnóstico do tumor.

de duração da doença foi maior naqueles com presença de neoplasias $(\mathrm{p}=0,006)$, conforme pode ser visto na Figura 1 .

O estudo da associação da presença de tumores com medicamentos utilizados para tratamento do LES pode ser encontrado na Tabela 3, onde se observa que nenhum deles influenciou na taxa de aparecimento de neoplasias.

Ao se comparar a incidência dos tumores mais comuns na amostra estudada com dados de projeção do INCA dos últimos 10 anos para mesma região ${ }^{12}$, sendo esta de 71/100.000 de tumores de mama em 10 anos e de 16/100.000 dos tumores de colo de útero em 10 anos, encontrou-se que pacientes com LES tinham uma incidência de tumores de colo do útero e de mama maior do que a
Tabela 2. Tumores encontrados na população com lúpus eritematoso sistêmico $(n=395)$

\begin{tabular}{|c|c|c|c|c|}
\hline & \multicolumn{2}{|c|}{$\begin{array}{c}\text { Amostra de pacientes com } \\
\text { lópus (tofal) } \\
n=395\end{array}$} & \multicolumn{2}{|c|}{$\begin{array}{l}\text { Amostra de pacientes com } \\
\text { lúpus e tumores } \\
n=22\end{array}$} \\
\hline & $n$ & $\%$ & $\mathrm{n}$ & $\%$ \\
\hline Colo do útero & 10 & 2,5 & 10 & 45,4 \\
\hline Mama & 9 & 2,2 & 9 & 40,9 \\
\hline Ovário & 1 & 0,2 & 1 & 4,5 \\
\hline Tireoide & 1 & 0,2 & 1 & 4,5 \\
\hline Ósseo & 1 & 0,2 & 1 & 4,5 \\
\hline Pele & 1 & 0,2 & 1 & 4,5 \\
\hline
\end{tabular}

Observação: uma paciente teve dois tumores.

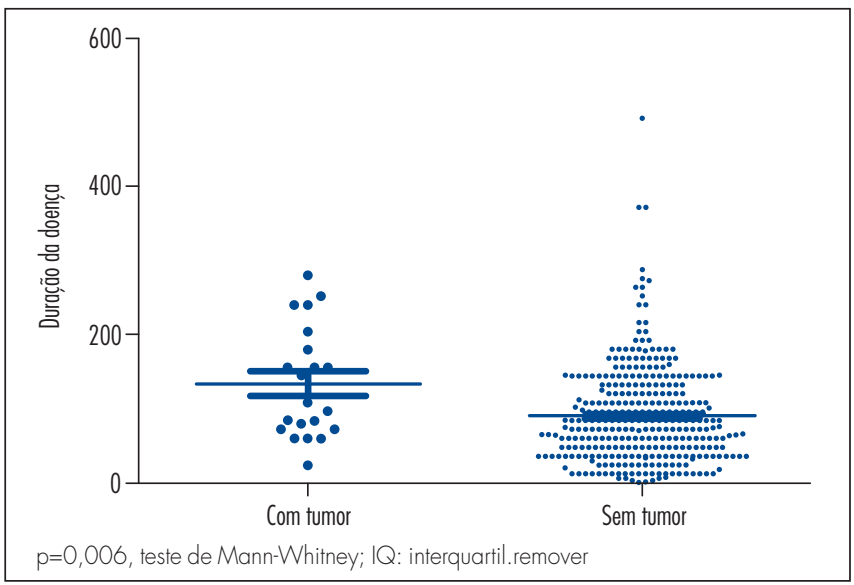

Figura 1. Comparação do tempo de duração da doença (em meses) em pacientes com tumores (mediana de 108 meses; intervalo interquartil 72,0-192,0 e sem tumor (mediana de 76 meses; intervalo interquartil 36,0-132,0); $p=0,006$, teste de Mann-Whitney.

Tabela 3. Estudos de associação de medicamentos utilizados para tratamento do lúpus eritematosos sistêmico com a presença de tumores

\begin{tabular}{lccccc}
\hline \multirow{2}{*}{ Medicamento } & \multicolumn{2}{c}{$\begin{array}{c}\text { Sem fumores } \\
\mathrm{n=373}\end{array}$} & \multicolumn{2}{c}{$\begin{array}{c}\text { Com fumores } \\
\mathrm{n=22}\end{array}$} & \multirow{2}{*}{ Valor $\mathrm{p}$} \\
\cline { 2 - 5 } & $\mathrm{n}$ & $\%$ & $\mathrm{n}$ & $\%$ & \\
\hline Azatioprina & 133 & 35,6 & 8 & 36,3 & 0,9 \\
Mefotrexafe & 74 & 19,8 & 7 & 31,8 & 0,1 \\
Ciclofosfamida & 94 & 25,2 & 4 & 18,1 & 0,6 \\
Glicocorticoides & 242 & 64,8 & 17 & 77,2 & 0,3 \\
Talidomida & 13 & 3,4 & 1 & 4,5 & 0,5 \\
\hline
\end{tabular}

população geral geral, sendo $\mathrm{p}<0,0001$ para ambos, com odds ratio (OR) de 10,4; intervalo de confiança de $95 \%$ (IC95\%) de 9,1-11,96 para neoplasia de colo do útero e $\mathrm{OR}=3,4$; IC95\% 3,1-3,7 para neoplasia de mama.

\section{Discussão}

O aumento no câncer de colo do útero em pacientes com LES é fato reconhecido na literatura ${ }^{4,5}$. Segundo Bernatsky et al. ${ }^{4}$ e Dreyer et al. ${ }^{5}$, esses pacientes não oferecem resistência contra a colonização pelo HPV. Acredita-se que isso ocorra secundariamente à imunodepressão causada pela própria doença e/ou pelo efeito imunossupressor 
dos medicamentos utilizados em seu tratamento, principalmente metotrexate e ciclofosfamida ${ }^{4,5}$. Em um estudo que incluiu pacientes brasileiras com LES, foi observado que essas tinham uma prevalência de infecções por HPV superior à dos controles $(20,2 \text { versus } 7,3 \%)^{13}$. Nesse mesmo estudo, os fatores de risco identificados foram o número de parceiros e uso de imunossupressores ${ }^{13}$. No presente trabalho, não se observou associação de neoplasias com quaisquer das drogas usadas pelas pacientes. Entretanto, o tempo de imunodepressão causada pela própria doença pode ter contribuído para a elevada prevalência de neoplasias encontrada, já que houve associação entre o seu aparecimento e a duração do LES. Defeitos na imunidade celular e na resposta imune inata têm sido associados ao câncer de colo do útero ${ }^{14,15}$. A resolução da infecção viral está, pelo menos parcialmente, na dependência da formação de anticorpos anti-HPV, recrutamento de macrófagos, células natural killer e de linfócitos T CD4 ativados $^{13}$. Essas funções estão prejudicadas em mulheres com LES ${ }^{13}$.

Apesar de a neoplasia de colo do útero ser considerada uma doença de países em desenvolvimento, no que se refere à sua ocorrência em pacientes com LES, essa diferença deixa de existir, havendo convergência da prevalência entre o Brasil e outros países desenvolvidos, como Dinamarca e América do Norte ${ }^{4,5}$.

A vacinação contra o HPV pode ser uma opção protetora para esse grupo populacional. Ela é considerada segura para uso em indivíduos imunodeprimidos, uma vez que não contém organismos vivos; o antígeno utilizado é formado por proteínas obtidas através de tecnologia recombinante e específicas para cada tipo de $\mathrm{HPV}^{16}$. Todavia, existe a possibilidade de que o uso de imunossupressores e/ou de corticoides prejudique a sua imunogenicidade ${ }^{13}$. Existem poucos dados acerca da eficácia da vacina contra o HPV em mulheres com lúpus até o momento. Um estudo incluindo 50 mulheres com LES e 50 controles, submetidas ao uso da vacina quadrivalente, demonstrou que ela é segura e bem tolerada e que conseguiu causar conversão para os 4 sorotipos injetados entre 78 e $95 \%$ nas lúpicas contra $80-98 \%$ nos controles ${ }^{17}$. Outra pesquisa, com 20 pacientes lúpicas entre 12 e 26 anos, usando a vacina quadrivalente, confirmou que essa vacina é bem tolerada e que não causa aumento de atividade do lúpus; a imunogenicidade demonstrada foi excelente, existindo soroconversão de $94 \%$ da amostra para os antígenos das 4 cepas inoculadas ${ }^{18}$. Pelo exposto, compreende-se que a vacinação contra o HPV é uma necessidade premente em pacientes com essa colagenose e que tais pacientes deveriam ser beneficiadas pelo sistema nacional de vacinação.

O câncer de mama tem sido descrito por outros autores como sendo menos comum entre as mulheres com lúpus do que na população em geral $^{4,8,19}$. A explicação oferecida é a de que o metabolismo dos estrógenos estaria alterado em pacientes com LES e que isso seria um fator protetor contra tumores dependentes desse hormônio ${ }^{4}$. Entretanto, existem argumentos contra tais afirmações. Um deles é o de que tem sido demonstrado que, em lúpus, os estrógenos atuam promovendo um aumento de função dos receptores de estrogênio (ER) $\alpha$ e não a sua diminuição ${ }^{19}$. Outro é o de que, segundo Tessier Cloutier et al..$^{20}$, são os tumores de mama ER negativos os que ocorrem menos frequentemente em pacientes com LES. Nossos resultados não confirmam o achado de uma menor prevalência de câncer de mama na população lúpica, demonstrando, ao contrário, o seu aumento. Tais achados necessitam ser confirmados por outras pesquisas. Uma justificativa para a discrepância entre os dados da literatura e os do estudo atual é a de que tanto o lúpus ${ }^{1}$ quanto o câncer de mama ${ }^{8}$ estão sujeitos a influências genéticas, podendo essa associação ter uma expressão diferente na população brasileira. Além disso, podem influir nessa variabilidade outros componentes tais como tipo de dieta ${ }^{21}$, obesidade ${ }^{22,23}$, níveis de vitamina $\mathrm{D}^{24,25}$ etc., os quais são peculiares a determinados grupos populacionais.

A natureza retrospectiva e a impossibilidade de se obter dados acerca da histologia dos tumores encontrados são algumas limitações do presente estudo. Pesquisas com amostragem superior, com desenho prospectivo e de pacientes acompanhadas por um período mais prolongado podem ajudar a esclarecer alguns aspectos dessa associação.

Concluindo, os autores ressaltam a necessidade de uma vigilância constante sobre aparecimentos de neoplasias de mama e de colo do útero em pacientes com LES. Isso pode ser conseguido pela conscientização de reumatologistas e ginecologistas acerca das associações aqui demonstradas, de medidas educativas para pacientes e de programas efetivos de rastreamento, os quais devem fazer parte do cuidado rotineiro oferecido a esse grupo de pacientes.

\section{Contribuição dos autores}

Thelma L. Skare: concepção do projeto, estudo estatístico, redação e revisão do texto.

Bruno V. Rocha: coleta de dados, revisão bibliográfica, redação e revisão do texto. 
1. Fortuna G, Brennan MT. Systemic lupus erythematosus: epidemiology, pathophysiology, manifestations, and management. Dent Clin North Am. 2013;57(4):631-55.

2. Pistiner M, Wallace DJ, Nessim S, Metzger AL, Klinenberg JR. Lupus erythematosus in the 1980s: a survey of 570 patients. Semin Arthritis Rheum. 1991;21(1):55-64.

3. Merrell M, Shulman LE. Determination of prognosis in chronic disease illustrated by systemic lupus erythematosus. J Chronic Dis. $1955 ; 1(1): 12-32$.

4. Bernatsky S, Kale M, Ramsey-Goldman R, Gordon C, Clarke AE. Systemic lupus and malignancies. Curr Opin Rheumatol. 2012;24(2):177-81.

5. Dreyer L, Faurschou M, Mogensen M, Jacobsen S. High incidence of potentially virus-induced malignancies in systemic lupus erythematosus: a long-term follow up study in a Danish cohort. Arthritis Rheum. $2011 ; 63(10): 3032-7$.

6. Hellstrom KE, Hellstrom I. Vaccines to treat cancer-an old approach whose time has arrived. J Cell Biochem. 2007;102(2):291-300.

7. Feng $Q$, Wei $H$, Morihara J, Stern J, Yu M, Kiviat $N$, et al. Th2 type inflammation promotes the gradual progression of HPV-infected cervical cells to cervical carcinoma. Gynecol Oncol. 2012;127(2):412-9.

8. Bernatsky S, Ramsey-Goldman R, Foulkes WD, Gordon C, Clarke AE. Breast, ovarian, and endometrial malignancies in systemic lupus erythematosus: a meta-analysis. Br J Cancer. 2011 ; 104(9): 1478-81 .

9. Dema B, Charles N. Advances in mechanisms of systemic lupus erythematosus. Discov Med. 2014;17(95):247-55.

10. de Souza VS, Santos RV. The emergence of human population genetics and narratives about the formation of the Brazilian nation (1950-1960). Stud Hist Philos Biol Biomed Sci. 2014 [ahead of print].

11. Hochberg MC. Updating the American College of Rheumatology revised criteria for systemic lupus erythematosus. Arthritis Rheum. 1997;40(9): 1725.

12. Brasil. Ministério da Saúde. Instituto Nacional de Câncer José de Alencar Gomes da Silva (INCA) [Internet]. Estimativa 2014: incidência de câncer no Brasil. Rio de Janeiro: INCA; 2014 [citado 2014 Abr 14]. Disponível em: <http://www.inca.gov.br/estimativa/2014/ estimativa-24042014.pdf>

13. Klumb EM, Pinto AC, Jesus GR, Araujo MJr, Jascone L, Gayer CR, et al. Are women with lupus at higher risk of HPV infection? Lupus. 2010;19(13):1485-91.
14. Padula SJ, Clark RB, Korn JH. Cell-mediated immunity in rheumatic disease. Hum Pathol. 1986;17(3):254-63.

15. Garred P, Voss A, Madsen HO, Junker P. Association of mannosebinding lectin gene variation with disease severity and infections in a population-based cohort of systemic lupus erythematosus patients. Genes Immun. 2001;2(8):442-50.

16. Macartney KK, Chiu C, Georgousakis M, Brotherton JM. Safety of human papillomavirus vaccines: a review. Drug Saf. 2013;36(6):393-412.

17. Mok CC, Ho LY, Fong LS, To $\mathrm{CH}$. Immunogenicity and safety of a quadrivalent human papillomavirus vaccine in patients with systemic lupus erythematosus: a case-control study. Ann Rheum Dis. 2013;72(5):659-64.

18. Soybilgic A, Onel KB, Utset T, Alexander K, Wagner-Weiner L. Safety and immunogenicity of the quadrivalent HPV vaccine in female systemic lupus erythematosus patients aged 12 to 26 years. Pediatric Rheumatol Online J [Internet]. 2013 [cited 2014 Jan 25];1 1:29. Available from: <http://www.ped-rheum.com/ content/pdf/1546-0096-11-29.pdf>

19. Liu HW, Lin HL, Yen JH, Tsai WC, Chiou SS, Chang JG, et al. Demethylation within the proximal promoter region of human estrogen receptor alpha gene correlates with its enhanced expression: implications for female bias in lupus. Mol Immunol. 2014;61(1):28-37.

20. Tessier Cloutier B, Clarke AE, Ramsey-Goldman R, Wang Y, Foulkes W, Gordon C, et al. Breast cancer in systemic lupus erythematosus. Oncology. 2013;85(2):117-21.

21. Colditz GA, Bohlke K, Berkey CS. Breast cancer risk accumulation starts early: prevention must also. Breast Cancer Res Treat. 2014;145(3):567-79.

22. Cecil DL, Park KH, Gad E, Childs JS, Higgins DM, Plymate SR, et al. T-helper I immunity, specific for the breast cancer antigen insulinlike growth factor-I receptor (IGF-IR), is associated with increased adiposity. Breast Cancer Res Treat. 2013;139(3):657-65.

23. Sinicato NA, Postal M, Peres FA, Peliçari KO, Marini R, dos Santos $A O$, et al. Obesity and cytokines in childhood-onset systemic lupus erythematosus. J Immunol Res. 2014;2014:162047.

24. Bidgoli SA, Azarshab H. Role of vitamin D deficiency and lack of sun exposure in the incidence of premenopausal breast cancer: a case control study in Sabzevar, Iran. Asian Pac J Cancer Prev. 2014;15(8):3391-6.

25. Feldman D, Krishnan AV, Swami S, Giovannucci E, Feldman BJ. The role of vitamin $D$ in reducing cancer risk and progression. Nat Rev Cancer. 2014;14(5):342-57. 